\title{
Coherent X-ray mirage: discovery and possible applications
}

\author{
Tatiana Pikuz ${ }^{1,2}$, Anatoly Faenov ${ }^{1,2,5}$, Sergey Magnitskiy $^{3}$, Nikolay Nagorskiy ${ }^{2}$, Momoko Tanaka ${ }^{1}$, \\ Masahiko Ishino ${ }^{1}$, Masaharu Nishikino ${ }^{1}$, Yuji Fukuda ${ }^{1}$, Masaki Kando ${ }^{1}$, Yoshiaki Kato ${ }^{4}$, and \\ Tetsuya Kawachi ${ }^{1}$ \\ ${ }^{1}$ Quantum Beam Science Directorate, Japan Atomic Energy Agency, Kizugawa, Kyoto 619-0215, Japan \\ ${ }^{2}$ Joint Institute for High Temperatures, Russian Academy of Sciences, Moscow 125412, Russia \\ ${ }^{3}$ International Laser Center of M.V. Lomonosov Moscow State University, Moscow, Russia \\ ${ }^{4}$ The Graduate School for the Creation of New Photonics Industries, Hamamatsu, Shizuoka 431-1202, Japan \\ ${ }^{5}$ Institute for Academic Initiatives, Osaka University, Suita, Osaka, 565-0871, Japan \\ (Received 18 February 2014; revised 31 March 2014; accepted 8 April 2014)
}

\begin{abstract}
In the far field of the intensity distribution of the beam delivered by a two-stage transient-collisional excitation $\mathrm{X}$-ray laser (XRL), a non-expected interference pattern that is stable from shot to shot has been discovered. It is demonstrated that the interference is caused by the emergence of an imaginary source in the amplifying plasma, which is phase matched to the radiation of the generator. The observed phenomenon is called an X-ray coherent mirage. To explain the obtained results, a new theoretical approach is developed. The basic essential conditions for formation of the $\mathrm{X}$-ray mirage are formulated, and possible applications are discussed. This paper details the experiments, including the formulation of the necessary and sufficient conditions for formation of the X-ray mirage, and possible applications are discussed.
\end{abstract}

Keywords: coherent seeded beams; mirage; phase-matching; X-ray lasers; X-ray plasma optics

\section{Introduction}

X-rays are a form of electromagnetic radiation with wavelengths shorter than the visible part of the spectrum. This means that practically all the phenomena existing in the optical, visible range should have their analogues in X-rays. However, while more than 100 years have passed since the discovery of X-rays, and despite the impressive achievements in X-ray physics since then, optical mirages ${ }^{[1]}$, an amazing and mysterious phenomenon of optical refraction, have never been observed in the $\mathrm{X}$-ray band. Indeed, the appearance of a mirage in the X-ray is extremely difficult because for all conventional materials the refractive indexes are close to 1 , and gradients of the refractive indexes are low in the $\mathrm{X}$-ray frequencies. Obviously, such properties practically eliminate refraction and the possibility of mirage formation in the X-ray spectral range. Nevertheless, recently a new unique medium has been created: laser-induced plasma,

Correspondence to: Tatiana Pikuz, Quantum Beam Science Directorate, Japan Atomic Energy Agency, Kizugawa, Kyoto 619-0215, Japan. Email: tapikuz@yahoo.com which has a noticeable X-ray refraction and is effective for $X$-ray laser $(X R L)$ generation ${ }^{[2-5]}$. After the first realization of an XRL, significant interest emerged in developing a coherent table-top XRL. In particular, a plasma-based transient collisional excitation $\mathrm{XRL}^{[6-9]}$ allowed one to effectively realize a compact coherent soft X-ray source. However, the coherence of the output radiation of a modern XRL is far from perfect, and the efforts of many research groups are aimed at improving both the temporal and the spatial coherence of XRLs.

Recently, using radiation from a plasma-based transient collisional excitation XRL ${ }^{[10]}$, the observation of an unknown interference structure in the spatial profile of the output beam of a two-stage plasma media XRL has been reported $^{[11]}$. Such a result led to founding and, after scrupulous theoretical analysis, to understanding this new mechanism, which could have a drastic influence on the spatial characteristics of XRL beams - the formation of X-ray coherent mirages $^{[11]}$, which are unique phenomena for the $\mathrm{X}$-ray spectral range. In this article, we present some new results and provide more detailed discussions about the $\mathrm{X}$-ray coherent mirage phenomenon and about its possible applications in science and technology. 
(a)

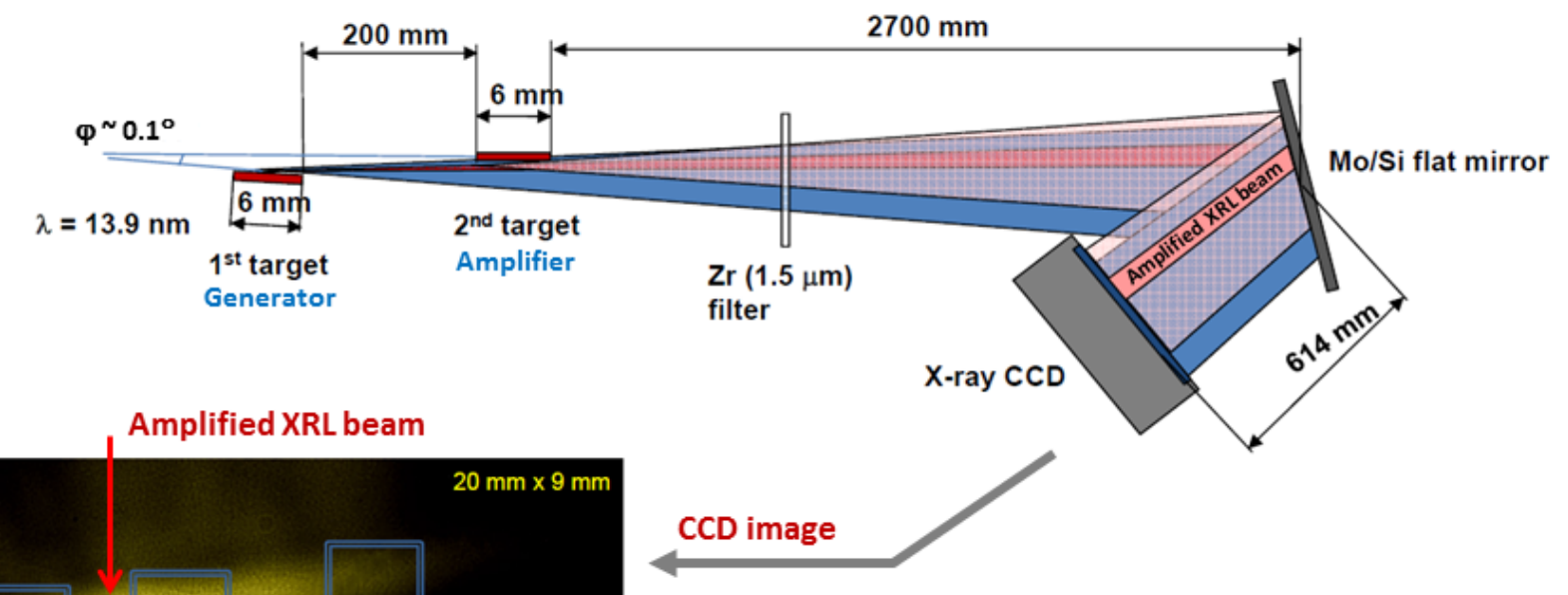

(b)
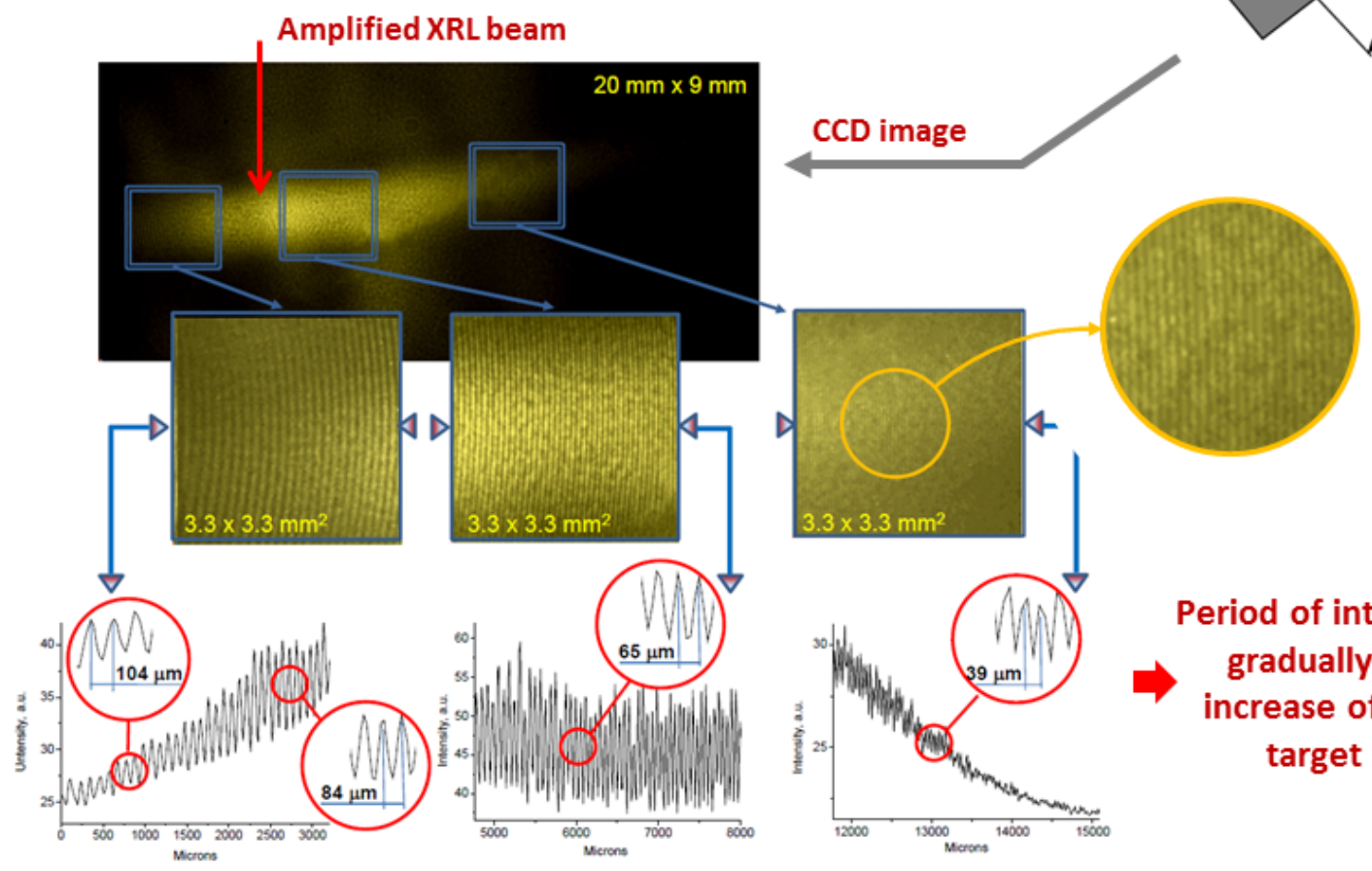

Period of interference fringes

gradually decreases on

increase of distance from

target of amplifier

Figure 1. (a) Scheme of the experiment. (b) Typical image of an XRL far-field intensity distribution, obtained in one shot.

\section{Experimental set-up and results}

The results presented here were obtained during an experimental study of the spatial characteristics of the far-field wave front of an XRL, built on a two-stage transientcollisional excitation oscillator-amplifier Ni-like Ag plasma $(\lambda=13.9 \mathrm{~nm})$ scheme $^{[10]}$, at Kansai Photon Science Institute of the Japan Atomic Energy Agency (Figure 1(a)). Thin silver foils for the oscillator and the amplifier were pumped, respectively, with the line-focused travelling wave laser pulses adjusted to the proper timing for effective amplification. The XRL pulse after the amplifier had energy of $\sim 300 \mathrm{~nJ}$, bandwidth narrower than $10^{-4}$, and pulse duration of $\sim 7 \mathrm{ps}$.

At specific conditions of XRL operation (delay between generator and amplifier pumping pulses $<10 \mathrm{ps}$ and relative angle between oscillator and amplifier targets $<0.6^{\circ}$ ), we found the appearance of an interference pattern (a sequence of dark and light rings) in the form of concentric rings in the output of the XRL beam. A typical image obtained by a back-illuminated X-ray charge-coupled device (CCD) with $2048 \times 2048$ pixels (the size of a pixel is $13.5 \mu \mathrm{m}$ ) at a distance of $3314 \mathrm{~mm}$ from the output of the XRL in one shot is shown in Figure 1(b). In the image, the bright spot elongated in the horizontal direction is a footprint of amplified XRL radiation. Concentric interference fringes are present over practically all of the beam profile. As can be clearly seen from the expanded insets of the image in Figure 1(b), the period of the fringes is gradually decreasing from the center to the peripheries. Such a structure was observed only in the case when two-stage XRL lasers were operated. This means that, in this case, the generator and the amplifier were turned on simultaneously. Special experiments, when the generator or amplifier was turned off, were also performed.

In the case of the output intensity distribution of the beam, such an interference pattern was never observed. Moreover, we checked the stability of such phenomenon appearing during XRL operation, and discovered that, under certain pumping parameters of the XRL, the ring structures were recorded consistently from shot to shot. 


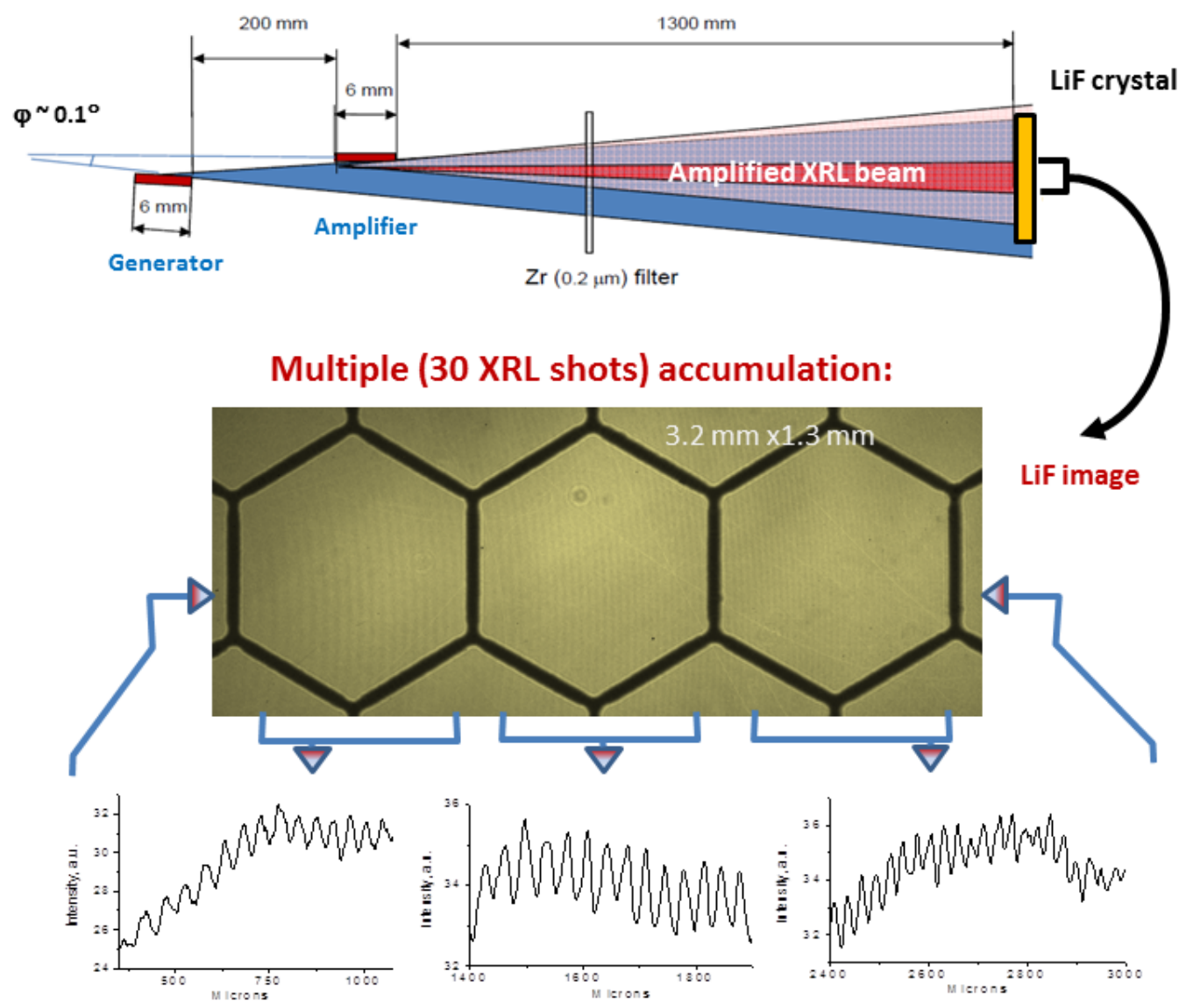

Figure 2. Experimental observation of the interference pattern in the intensity distribution of the output of an oscillator-amplifier XRL beam after 30 accumulated shots.

Such high stability of generation of interference fringes allowed us to provide an additional series of experiments. A LiF crystal detector ${ }^{[12-14]}$ with submicron spatial resolution was used for measurements of the spatial-intensity distribution of the XRL beam, which were averaged over 30 laser shots. The scheme of the experiment and a typical image are presented in Figure 2. Our measurement indicates high stability of the interference patterns, which appears despite the accumulation of a large number of XRL shots. This gives us the possibility to conclude that the observed phenomenon has a fundamental character; both sources are rigidly phased and do not significantly depend on fluctuating plasma parameters.

\section{Discussion}

The appearance of such a concentric interference pattern in the spatial profile of the output beam is a rather unexpected observation, because there are no apparent physical causes that can lead to it. Indeed, according to optical interferometry fundamentals, the observation of circular concentric fringes indicates an existence of two point coherent sources, separated in space, located on the axis, passing through the center of the circles (see Figure 3). This means that detected rings are produced by an interference of radiation emitted by two coherent point sources, which were produced in our experimental conditions. Obviously the first source is located in the XRL generator (see Figure 1(a) and Figure 2). To determine the position of the second source, we calculated interference patterns, and by matching their period with the period of experimentally observed interference patterns (see Figure 4) we found that second source must be located at the distance of $203 \pm 2 \mathrm{~mm}$ from the first one. From the layout of the XRL system (see Figure 1(a) and Figure 2) it follows that the second source should be located somewhere in the middle of the amplifying plasma. In fact, the distance between the generator and the amplifier targets is $200 \mathrm{~mm}$, and the length of both targets is $6 \mathrm{~mm}$. So the distance $203 \mathrm{~mm}$ is the distance from the exit of generator to the center of the amplifier. Although this geometrical consideration is quite simple, it is not easy to find physical reasons for the emergence of the second point source in the amplifying plasma. Moreover, to be able to interfere with the source from the generator, the second source should be phase matched to the first one.

Based on our observations, we undertook theoretical analyses, which showed that the user of the standard system of Maxwell-Bloch equations, typically describing amplification of seeded beams in plasma media, could not explain 


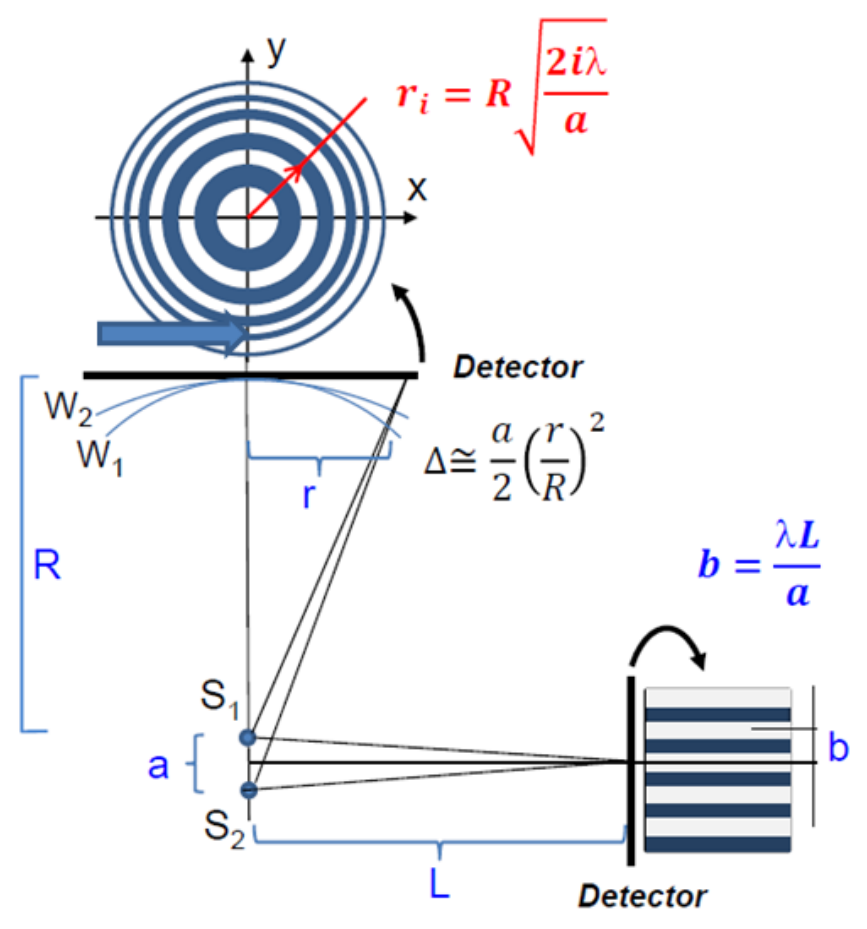

$S_{1}$ and $S_{2}-$ coherent sources

Figure 3. Optical scheme of fringe formation by two point coherent sources.

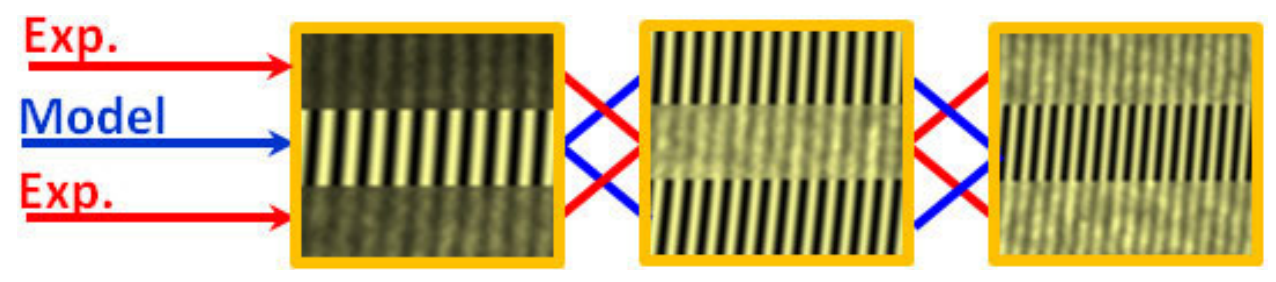

Figure 4. Comparisons of experimental and modeled interference patterns for the case of the distance between two phase-matched X-ray point sources being $203 \mathrm{~mm}$.

the emergence of the second source and the observed phenomenon. Indeed, from our experiments we could see that the phase front of the stimulated emission should repeat the phase front of an initial wave. This means that the amplified seeded X-ray beam could not create a spherical wave with a phase front of another curvature. This led to the necessity of developing a new theoretical approach. Such an approach is proposed in ${ }^{[11]}$ and is based on the fact that in our experiments (due to the high density and strong localization of laser-produced plasma media in space) the Xray beam propagation and transformation are similar to the phenomenon of classical mirages in a strongly inhomogeneous atmosphere.

It is supposed in ${ }^{[11]}$ that, mathematically, a mirage is a part of the total radiation, which remains after the initial radiation is subtracted from it, under the assumption that the initial radiation propagates through a medium without distortion in the medium. We took to the account that the complex amplitude of the output XRL beam $A(x, y, z)$ consisted of two terms: the amplitude of the incident beam propagating in empty space $A_{i n c}(x, y, z)$ and the amplitude of mirage radiation emerging in the plasma $A_{M}(x, y, z)$ :

$$
A(x, y, z)=A_{\text {inc }}(x, y, z)+A_{M}(x, y, z) .
$$

To determine which imaginary source corresponds to the output mirage radiation $A_{M}^{i m}(x ; y ; z)$, it is enough to solve the equation $A_{M}(x, y, z)=A(x, y, z)-A_{i n c}(x, y, z)$ for the output radiation and to run the complex amplitude $A_{M}(x, y, z)$ backward in empty space. Schematically, the algorithm is shown in Figure 5.

Suppose that a wide divergent X-ray beam is incident on a small piece of plasma medium. Several factors will influence the formation of a mirage. First of all, the spatial distribution of the refractive index, but also the gain of the medium, will play a significant role in the process. Besides, since in typical $\mathrm{XRL}$ the gain coefficient is very high, it is considered to be the effects of saturation in signal amplification. Under 
(a)

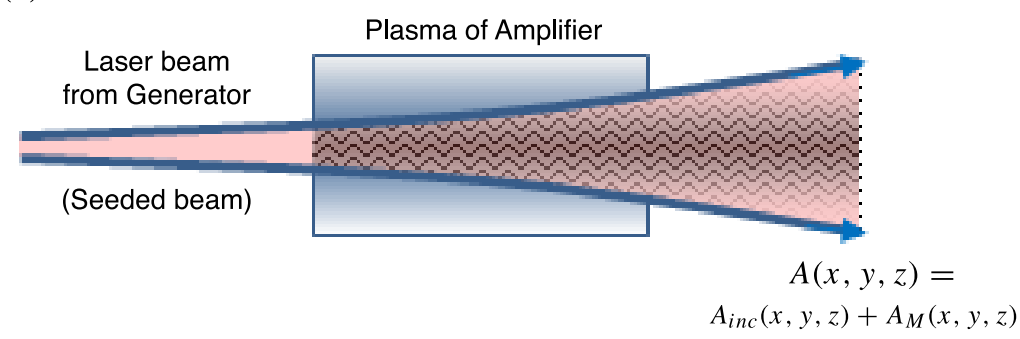

(b)

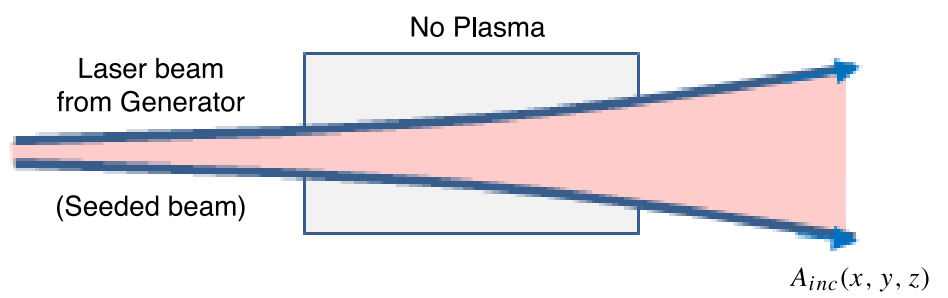

(c)

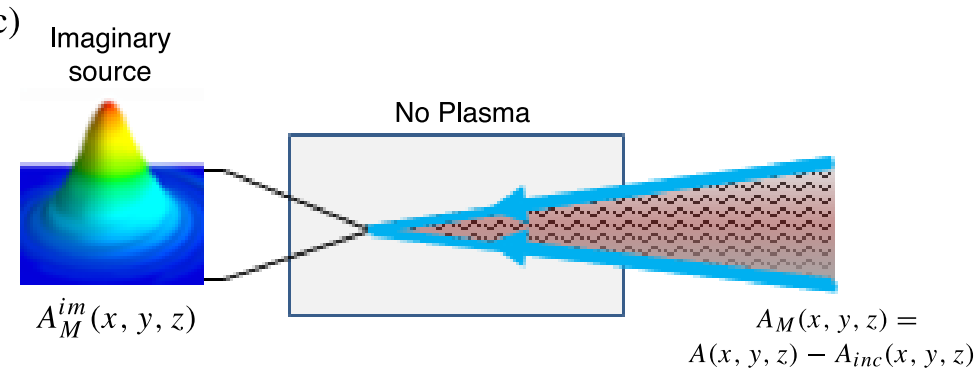

Figure 5. Schematic explanation of the theoretical algorithm for X-ray mirage formation. (a) XRL radiation from the generator (seeded beam) propagated in empty space with complex amplitude $A_{i n c}(x, y, z)$; (b) after propagation of the seeded beam through plasma of the amplifier, the complex amplitude of the distorted radiation $A(x, y, z)$ consists of two terms: the complex amplitude $A_{i n c}(x, y, z)$ and the complex amplitude of the mirage $A_{M}(x, y, z)$; (c) formation of imaginary source $A_{M^{i m}}(x, y, z)$ by running the complex amplitude $A_{M}(x, y, z)$ backward in empty space.

these assumptions, the optical properties of the plasma will be similar to the optical properties of some effective inhomogeneous lens, where the thickness of the lens imitates the refractive index of the plasma. This means that the lower the refractive index, the thicker the lens. In the case when a seeded beam passes through such an optical system, it will consist of a set of outgoing waves generated by either a point or an extended imaginary source located along the axis of symmetry. If the plasma has a gain, the central part of the beam will be predominantly amplified. Since the electron density $n_{\mathrm{e}}(x ; y)$ surface is approximated by a paraboloid, the central part of the plasma works as an ideal aberration-free lens. From such a consideration it follows that the higher the gain, the narrower the axial region of the plasma which contributes to the beam amplification. This leads to the conclusion that an emerged virtual source will be closer to a point one and, evidently, will be phased with the incident radiation. The numerical simulations showed that, for the typical parameters of XRL plasma media, a coherent mirage in the form of an imaginary point source arises. It is found that the position of this point source in space is in accordance with our obtained experimental data.
The development of the theory and carrying out of the modelling allowed us to formulate the necessary and sufficient conditions for formation of an X-ray mirage in plasma media: (1) there should be a local maximum in the $N_{\mathrm{e}}(x ; y)$ distribution, (2) the spatial location of smallsignal of gain distribution $G(x, y)$ in the plane $x, y$, which is perpendicular to the XRL beam propagation should coincide with the position of the local maximum of $N_{\mathrm{e}}(x ; y)$, the (3) spatial size of the gain region should be equal to or less than the width of the $N_{\mathrm{e}}(x ; y)$ local maximum, and (4) the uniformity of the plasma along the direction of beam propagation should be sufficiently high.

It is clear that these four conditions are difficult to reach in experiments. On the other hand, each of these conditions in itself is not unique to a plasma induced by short laser pulses on the surface of solid targets and correspondent to the conditions, which is usually necessary for optimal transient-collisional XRL generation. This means that $\mathrm{X}$-ray radiation amplification in mirage formation is the key issue. If there is no gain, the whole area of plasma filled with radiation would participate in the process of beam formation. As a result, output radiation with non-uniform intensity and with a sufficiently inhomogeneous phase front 
would be formed. But this is not a mirage. As not in every inhomogeneous atmosphere does an optical illusion occur, similarly not in every inhomogeneous plasma does an X-ray mirage occur.

\section{Conclusions}

The experimental observation of an X-ray coherent mirage in a two-stage XRL system, and a theoretical approach, which explained a mechanism of that phenomenon, are discussed. It is shown that, in XRL plasma media, both refraction and diffraction accompany the process of amplification and play significant roles in the emergence of the virtual source of the mirage. There are various possibilities of how the Xray mirage phenomenon could be useful for further improvement of XRL systems and in developing new advanced methods of plasma media diagnostics. The discovery of $\mathrm{X}$-ray mirages could be applied to further develop modern $\mathrm{X}$-ray technology, for example, for generation of adaptively controlled phased X-ray beams and plasma X-ray optics.

\section{Acknowledgement}

This work was partly supported by RFBR-JSPS collaboration program (RFBR Grant 14-02-92107).

\section{References}

1. A. A. Knowlton, Science 50, 328 (1919).

2. R. C. Elton, X-ray Lasers (Academic, 1990).
3. H. Daido, Rep. Progr. Phys. 65, 1513 (2002).

4. P. Jaegle, Coherent Sources of XUV Radiation (Springer, 2006).

5. S. Suckewer and P. Jaegle, Laser Phys. Lett. 6, 411 (2009).

6. P. V. Nickles, V. N. Shlyaptsev, M. Kalachnikov, M. Schnürer, I. Will, and W. Sandner, Phys. Rev. Lett. 78, 2748 (1997).

7. J. Dunn, A. L. Osterheld, R. Shepherd, W. E. White, V. N. Shlyaptsev, and R. E. Stewart, Phys. Rev. Lett. 80, 2825 (1998).

8. T. Kawachi, M. Kado, M. Tanaka, A. Sasaki, N. Hasegawa, A. V. Kilpio, S. Namba, K. Nagashima, P. Lu, K. Takahashi, H. Tang, R. Tai, M. Kishimoto, M. Koike, H. Daido, and Y. Kato, Phys. Rev. A. 66, 033815 (2002).

9. B. A. Reagan, W. Li, L. Urbanski, K. A. Wernsing, C. Salsbury, C. Baumgarten, M. C. Marconi, C. S. Menoni, and J. J. Rocca, Opt. Express 21, 28380 (2013).

10. M. Nishikino, N. Hasegawa, T. Kawachi, H. Yamatani, K. Sukegawa, and K. Nagashima, Appl. Opt. 47, 1129 (2008).

11. S. Magnitskiy, N. Nagorskiy, A. Faenov, T. Pikuz, T. Tanaka, M. Ishino, M. Nishikino, M. Fukuda, M. Kando, T. Kawachi, and Y. Kato, Nature Commun. 4, 2923 (2013).

12. A. Ya. Faenov, Y. Kato, M. Tanaka, T. A. Pikuz, M. Kishimoto, M. Ishino, M. Nishikino, Y. Fukuda, S. V. Bulanov, and T. Kawachi, Opt. Lett. 34, 941 (2009).

13. T. Pikuz, A. Faenov, Y. Fukuda, M. Kando, P. Bolton, A. Mitrofanov, A. Vinogradov, M. Nagasono, H. Ohashi, M. Yabashi, K. Tono, Y. Senba, T. Togashi, and T. Ishikawa, Opt. Express 20, 3424 (2012).

14. T. A. Pikuz, A. Ya. Faenov, Y. Fukuda, M. Kando, P. Bolton, A. Mitrofanov, A. V. Vinogradov, M. Nagasono, H. Ohashi, M. Yabashi, K. Tono, Y. Senba, T. Togashi, and T. Ishikawa, Appl. Opt. 52, 509 (2013). 